\title{
Update on the Clinical Effect of Acupuncture Therapy in Patients with Gouty Arthritis: Systematic Review and Meta-Analysis
}

\author{
Wei-wei Lu, Jin-ming Zhang, Zheng-tao Lv, and An-min Chen \\ Department of Orthopedics, Tongji Hospital, Tongji Medical College, Huazhong University of Science and Technology, \\ Wuhan, Hubei 430030, China
}

Correspondence should be addressed to An-min Chen; anminchen@hust.edu.cn

Received 17 May 2016; Revised 1 August 2016; Accepted 24 August 2016

Academic Editor: Deborah A. Kennedy

Copyright (C) 2016 Wei-wei Lu et al. This is an open access article distributed under the Creative Commons Attribution License, which permits unrestricted use, distribution, and reproduction in any medium, provided the original work is properly cited.

Objective. The aim of this study is to evaluate the clinical efficacy and safety of acupuncture therapy in the treatment of acute gouty arthritis. Methods. A literature search of PubMed, EMBASE, ISI Web of Science, CENTRAL, and CNKI was conducted from the inception date of each database up to October 2015. Two investigators screened each article independently and were blinded to the findings of the other reviewer. Data was extracted according to the predetermined collection form. Meta-analysis was performed. Results. We analyzed data from 28 RCTs involving 2237 patients with gouty arthritis. Compared with conventional pharmacological treatments acupuncture was more effective in rendering patients free from symptoms after 24 hours, lowering serum urate, alleviating pain associated with gouty arthritis, and decreasing the ESR; regarding CRP, no statistically significant difference was found. In addition, the frequency of adverse events in acupuncture treatment was lower than that in control group. Conclusion. Based on the findings of our study, we cautiously suggest that acupuncture is an effective and safe therapy for patients with gouty arthritis. However, the potential beneficial effect of acupuncture might be overstated due to the methodological deficiency of included studies. High quality RCTs with larger scale are encouraged.

\section{Introduction}

Gouty arthritis is a disorder of purine metabolism and results from monosodium urate (MSU) crystal deposition in and around the joints caused by longstanding hyperuricaemia, which is defined as a serum uric acid (sUA) concentration exceeding the limit of sUA solubility $(6.8 \mathrm{mg} / \mathrm{dL})[1,2]$. This chronic inflammatory condition is mediated by MSU crystals and characterized by recurrent attacks of monoarthritis or polyarthritis [3]. Gouty arthritis is the most prevalent inflammatory arthritis in developed countries, especially in elder men; and the incidence was reported to increase over the past 20 years [4]. Quality of life is impaired during intercritical periods and during flares in addition to associated comorbidities such as obesity, diabetes mellitus, hypertension, hyperlipidaemia, and chronic renal disease [5-7]. Without proper treatment, acute gouty arthritis can progress into a chronic, deforming, and physically disabling disease through the development of tophi, joint destruction, and persistent pain $[8,9]$.

The American College of Rheumatology (ACR) and European League Against Rheumatism (EULAR) guidelines for the pharmacologic management of acute gouty arthritis include oral colchicine, nonsteroidal anti-inflammatory drugs (NSAIDs), and corticosteroids [10, 11]. However, the side effects associated with pharmacological agents restrict their long-term use. Some authors have pointed out that management of gouty arthritis is often suboptimal and that flares are inevitable, necessitating better strategies to handle attacks [12].

Complementary and alternative medicine (CAM) is widely advocated to face the increasing demand for nonpharmacological approaches. As a mainstream of CAM therapy, acupuncture based on traditional Chinese medicine (TCM) theory has been commonly used for the treatment of gouty arthritis in Chinese cultures. A previous systematic review 
had reported the clinical effect of acupuncture in relieving pain and decreasing serum uric acid, whereas only ten RCTs were included, the heterogeneity among studies was not discussed, and the security of acupuncture therapy was not confirmed [13]. Recently, new literatures to evaluate the efficacy and safety of acupuncture therapy for gouty arthritis are emerging. Therefore, an updated meta-analysis is required.

\section{Methods}

This systematic review was conducted in accordance with the Preferred Reporting Items for Systematic Reviews and MetaAnalyses (PRISMA) guidelines [14].

2.1. Search Strategy. A comprehensive literature search of PubMed, EMBASE, CENTRAL, ISI Web of Science, and CNKI was conducted. All the above databases were searched from their inception dates up to the latest issue (October 2015), without language restriction. Medical subject headings $(\mathrm{MeSH})$ and free text words were combined to retrieve all the potential studies. MeSH were modified based on the specifications of each database. The following search strategy was used for the literature search in PubMed, CENTRAL, and ISI Web of Science: ("Gout" or Gout or gouts) and ("Acupuncture Therapy" or acupuncture or moxibustion or acupoint or acupressure or acustimulation). For CNKI, search terms were "Zhen" and "Tongfeng". In addition, the bibliographies of relevant systematic reviews and clinical guidelines were manually searched. The reference section for each study was also searched.

2.2. Types of Participants. To be included in our systematic review, the enrolled subjects had to be diagnosed as gouty arthritis according to ascertained diagnostic criteria: European League Against Rheumatism (EULAR) criteria, the American Rheumatism Association (ARA) preliminary classification criteria for acute gout 1977 [15], Mexico 2010 [16], Netherland 2010 [17], or the Criteria of Diagnosis and Therapeutic Effect of Diseases and Syndromes in TCM issued by the State Administration of TCM [18]. No restrictions on race, age, and sex were imposed.

2.3. Interventions. Patients in experimental groups mainly received acupuncture therapy (including manual acupuncture and electroacupuncture), either alone or in combination with pharmacological treatment or TCM treatment, without differentiating different acupuncture techniques, acupoints selection, or needle materials. Patients in control groups were treated with western medicine (including colchicine, allopurinol, indomethacin, benzbromarone, celecoxib, probenecid, meloxicam, and ibuprofen); no specific types of drugs were imposed.

2.4. Outcome Measurements. The primary outcome measure was clinical effect and the frequency of adverse events in both groups; the secondary outcome measurements included clinical parameters associated with acute gouty arthritis, such as serum uric acid, pain intensity using visual analogue scale
(VAS), erythrocyte sedimentation rate (ESR), and C-reactive protein (CRP).

2.5. Types of Publication. The included studies were required to be randomized controlled trials aiming to assess the efficacy of acupuncture therapy for gouty arthritis. Articles regarding animal experiments, reviews articles, case reports, or expert experience reports were excluded.

2.6. Data Extraction. Two investigators (Wei-wei Lu and Jinming Zhang) screened each article independently and were blinded to the findings of the other reviewer. According to the predetermined inclusion criteria, two reviewers performed strict screening to identify qualified articles independently, and they extracted data from these eligible articles using a standardized data collection form, which included first author, year of the publication, study design, baseline characteristics for participants in different groups, diagnostic criteria of gouty arthritis, interventions and control treatment, main outcome assessments, duration of treatments, and adverse events.

Any disagreement between the two reviewers was resolved through discussion until a consensus was reached. The third review author (Jin-ming Zhang) was consulted if a consensus could not be reached.

2.7. Quality Assessment. Cochrane Collaboration's tool was utilized to assess the risk of bias in the selected RCTs, which was based on seven items: random sequence generation, allocation concealment, blinding of participants and personnel, blinding of outcome assessment, incomplete outcome data, selective reporting, and other sources of bias [19]. Two reviewers assessed the risk of bias among studies independently; the results were compared afterwards. Disagreements regarding the risk of bias assessment were settled by discussion and consensus between reviewers.

2.8. Data Synthesis and Analysis. The enrolled participants were dichotomized into being cured and not cured to express the intervention effect. Odds ratio (OR) and the associated 95\% confidence intervals (CIs) were calculated for clinical effect and frequency of adverse events. The mean difference (MD) for changes from baseline in the continuous variables was calculated using the same methodology. Random effect model was employed for meta-analysis, since the homogeneity of the included studies could not be guaranteed. The chi-squared test and the Higgins $I^{2}$ test were used to assess the heterogeneity among studies $\left(P>0.1\right.$ and $I^{2}$ indicate acceptable heterogeneity). Forest plot and funnel plot were generated via RevMan 5.3 (Copenhagen: the Nordic Cochrane Centre, the Cochrane Collaboration, 2014).

Metaregression was performed using Stata version 12.0 (StataCorp LP, USA) to find the possible source of heterogeneity, such as acupuncture type, treatment duration, and whether combined with other therapy. Begg's rank correlation test and Egger's linear regression test were used to evaluate the publication bias if the number of included studies was greater than ten. 


\section{Results}

3.1. Literature Search Results. An initial search of RCTs yielded 379 potential literature citations, including 27 from PubMed, 8 from CENTRAL, 57 from EMBASE, 30 from ISI Web of Science, 255 from CNKI, and additional two records from other sources. 47 articles were deleted because they were duplicates for retrieving. According to the prespecified inclusion criteria, 66 potentially relevant studies were selected and retrieved for a full-text assessment after reading their titles and abstracts. Of the remaining 66 studies, two were deleted because they were non-RCTs, two were duplicates for publication, one study did not provide available data, and 33 studies employed uncomfortable intervention. A total of 28 studies [20-47] were deemed eligible for inclusion in this review. The literature screening process is presented in a flowchart in Figure 1.

3.2. Study Characteristics. The main characteristics of included studies are summarized in Table 1 . The 28 studies included a total of 1174 patients in acupuncture group and 1063 patients in control group. Subjects were diagnosed as gouty arthritis according to either the criteria stipulated by US Rheumatology Association or the criteria stipulated by State Administration of TCM. The age of enrolled subjects ranged from 18 to 80 years. All the studies were conducted by Chinese investigators in a single center and published between 2002 and 2015. Clinical efficacy of acupuncture was evaluated by all studies, but only ten of them analyzed the safety in both groups.

Patients in acupuncture group received manual acupuncture or electroacupuncture; the acupoints selection was based on TCM meridian theory. The acupuncture was applied alone or in combination with other treatment, such as Chinese herbal medicine, acupoint injection, and local blocking therapy. Acupuncture therapy was administered daily or every two days, the needles were retained for 20 to 30 minutes for each session, and the treatment course ranged from 5 days to 28 days. The detailed information is listed in Table 2.

3.3. Quality Assessment. The risk of bias among studies was assessed using Cochrane Collaboration's tool. All studies included the suggested randomization, half of the studies $[22,28,29,34-38,41-43,45-47]$ reported the method of random sequence generation, and five studies [24, 27, $30,31,39$ ] were judged to high risk of bias because the patients were arranged according to their registration order. No study reported detail about allocation concealment. The blinding of outcome assessment was judged to unclear risk of bias because no studies mentioned blinding of outcome assessment. The blinding of participants and personnel was judged to high risk of bias as it was impossible to carry out in our included studies. When it comes to incomplete data, only four studies $[27,34,41,47]$ provided the number of dropouts and reason for withdrawal. All the studies reported the prespecified outcome measurements. Baseline similarities seemed to be achieved by each study: no statistical differences were detected in age, gender, or symptom duration. Five studies [24, 27, 30, 31, 39] were judged to high risk of bias while the other 23 were unclear risk of bias. Judgements about each risk of bias item for each included study were summarized in Figures 2 and 3.

3.4. Clinical Effect. All the included studies employed clinical effect as outcome assessment; the therapeutic effect was evaluated in accordance with the Criteria of Diagnosis and Therapeutic Effect of Diseases and Syndromes in TCM issued by the State Administration of TCM in 1994 [18]. The patients were defined as complete resolution of acute attack when the joints swelling, pain, and redness disappeared with normal laboratory indices 24 hours after the treatment, improved when symptoms and signs alleviated with laboratory indices improved, or failed when the symptoms and laboratory indices did not have improvement. Thus, the patients in experimental and control groups were dichotomized as complete resolution and not completely resolved; OR and 95\% CI in each study were calculated based upon the raw data. The combined effects of 28 individual studies showed that acupuncture therapy could further improve the clinical cure rate compared with western medicine (OR 2.71; 95\% CI 2.22, 3.32; $P<0.00001)$; the results of heterogeneity test indicated no obvious heterogeneity $\left(P=0.24, I^{2}=15 \%\right)$ (Figure 4$)$.

3.5. Uric Acid. Twenty-two studies [20, 25-39, 41-43, 45-47] measured blood uric acid as outcome; changes from baseline were calculated. Data extracted from 22 individual studies showed that heterogeneity existed $\left(P<0.00001, I^{2}=85.1 \%\right)$; random effects model was utilized for statistical analysis. Pooled data suggested that acupuncture therapy could further decrease uric acid than western pharmacological treatment (MD 41.30; 95\% CI 24.86, 57.74; $P<0.00001$ ) (Figure 5).

3.6. Pain Intensity (VAS Score). Seven studies $[29,33,34,41$, $43,46,47]$ that measured pain intensity using VAS score were identified. Data extracted showed obvious heterogeneity in the consistency of study results $\left(P<0.00001, I^{2}=\right.$ 94\%); random effects model was employed. Among the seven studies, six found that acupuncture therapy could further improve the VAS score than pharmacological therapy, whereas Liu et al. reported an opposite result. The combination of results showed that acupuncture could further improve pain associated with acute gouty arthritis (MD 1.92; 95\% CI 0.96, 2.87; $P<0.0001$ ) (Figure 6).

3.7. ESR. Five studies $[25,30,33,34,43]$ reported on ESR; only one study found statistically significant difference regarding the decrease of ESR in acupuncture group and control group. The heterogeneity was acceptable $(P=0.25$, $\left.I^{2}=26 \%\right)$; the pooled data showed that acupuncture therapy was better than western medicine in decreasing ESR (MD $1.75 ; 95 \%$ CI 0.11, 3.38; $P=0.04$ ) (Figure 7).

3.8. CRP. Four studies $[25,30,33,34]$ measured CRP as outcome; statistically significant difference was detected in only one study. No obvious heterogeneity was found $(P=$ $0.23, I^{2}=30 \%$ ); the combined data showed no significant difference in decreasing CRP between acupuncture and 


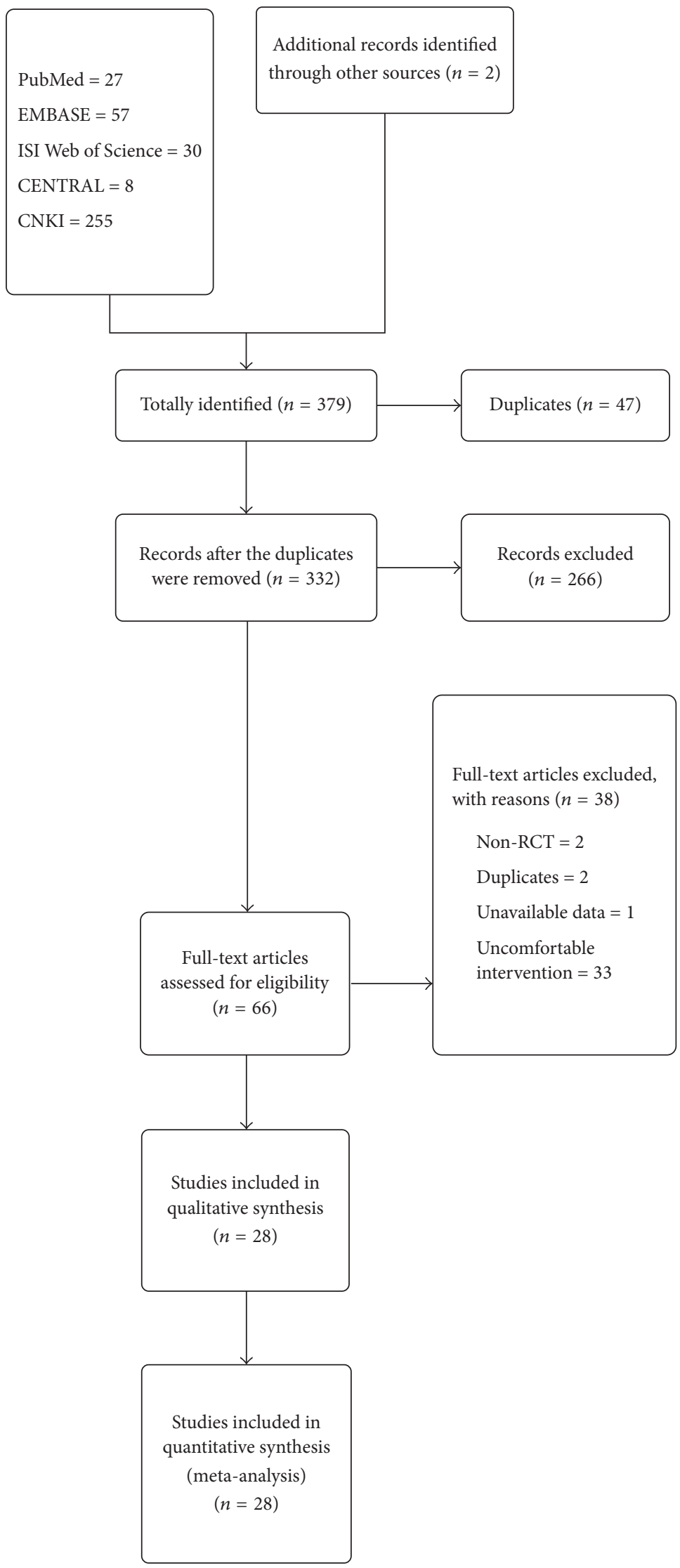

FIGURE 1: Flow diagram of the literature selection. 


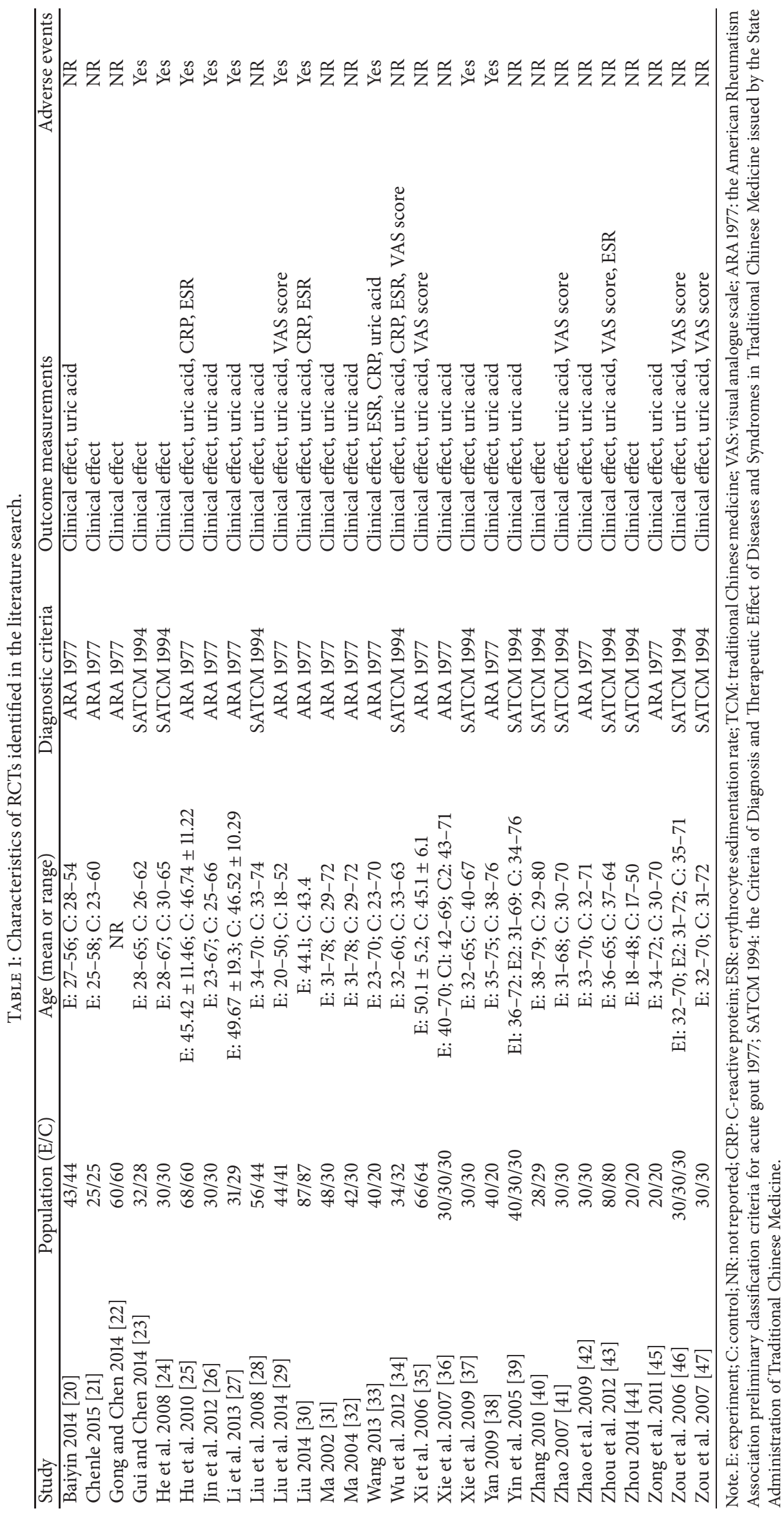




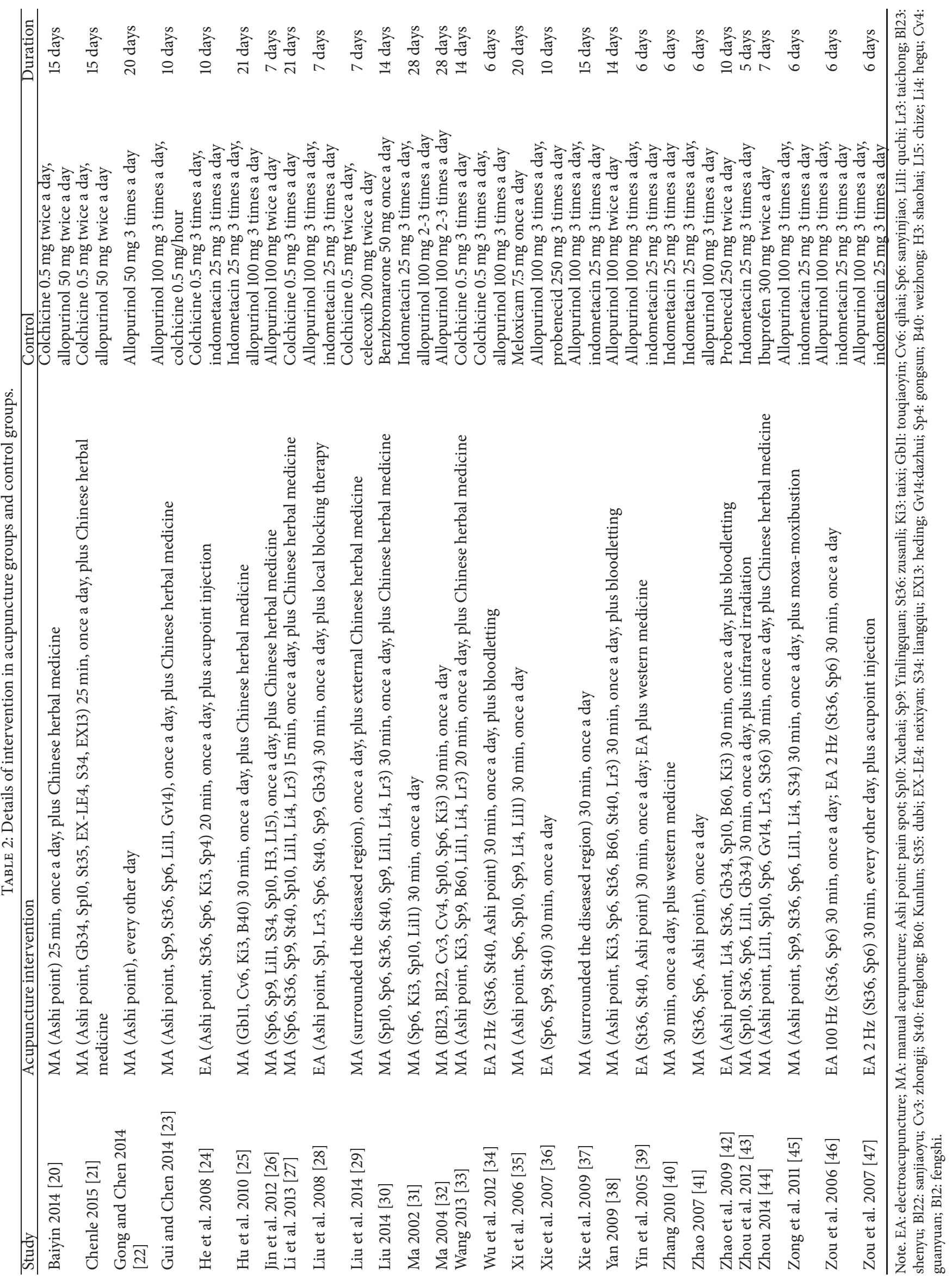




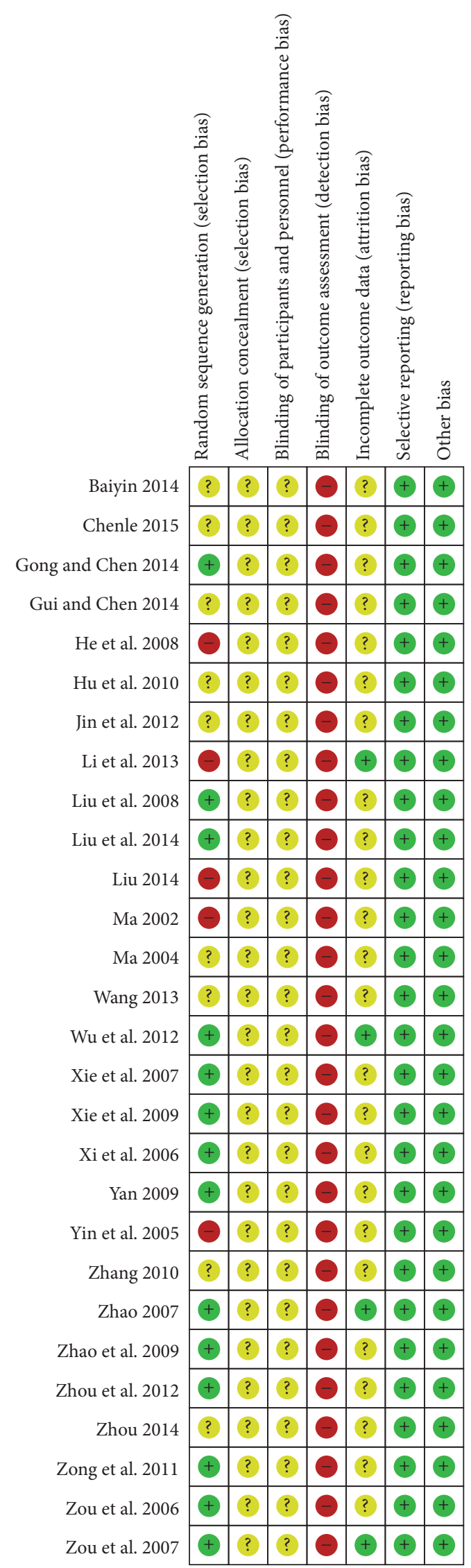

FIGURE 2: Risk of bias summary: review authors' judgements about each risk of bias item for each included study. 


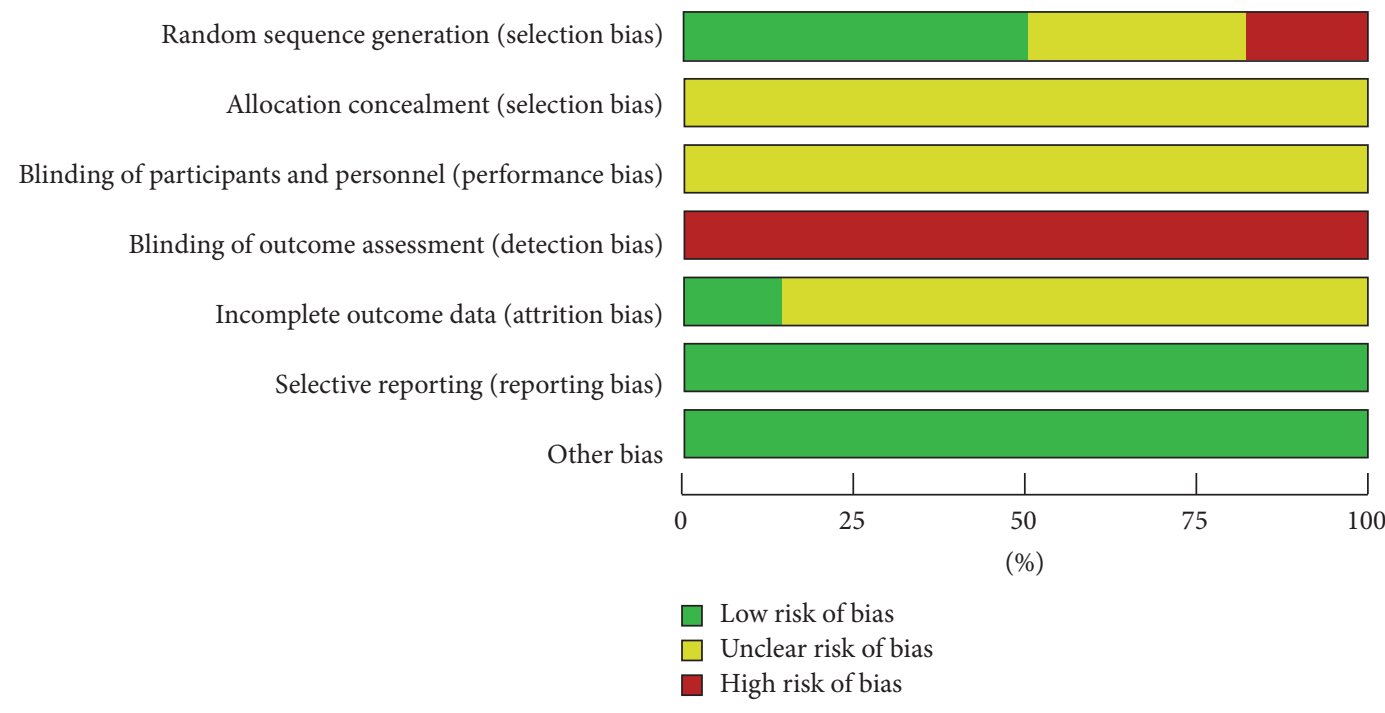

FIGURE 3: Risk of bias graph: review authors' judgements about each risk of bias item presented as percentages across all included studies.

control therapy $(\mathrm{MD}-0.26 ; 95 \% \mathrm{CI}-1.42,0.90 ; P=0.66)$ (Figure 8).

3.9. Adverse Events. Among the included studies, ten [23$27,29,30,33,37,38]$ reported adverse events associated with acupuncture or western medicine. The reported adverse events mainly included gastrointestinal tract reaction, central nervous system reaction, leukopenia, skin rash, and fainting during acupuncture treatment. Except two studies [26, 38], eight studies found a higher prevalence of adverse events in the control group than that in the acupuncture group. The pooled OR showed a statistically significant lower risk of adverse events in acupuncture group when compared with western medicine (OR 0.08, 95\% CI 0.03, 0.23; $I^{2}=34 \%$ ) (Figure 9).

3.10. Metaregression. Metaregression was conducted by residual (restricted) maximum likelihood (REML) with Knapp-Hartung modification. Three possible factors that may contribute to heterogeneity among studies were tested: acupuncture type (manual acupuncture or electroacupuncture), combined therapy (whether the patients were treated with acupuncture alone or combined with other treatment), and duration of treatment (within a week or longer than a week). The results of metaregression were listed in Table 3. Combined therapy and duration of treatment were not statistically correlated with the heterogeneity in uric acid or VAS score. Acupuncture type administered could explain $20.20 \%$ and $23.55 \%$ of the heterogeneity in uric acid and VAS score, respectively. However, the correspondence between acupuncture type and heterogeneity within VAS score did not reach a statistical difference.

3.11. Publication Bias. Publication biases were presented by funnel plots (Figures 10 and 11), and the resulting graphs show no obvious asymmetry for clinical effect and uric acid. Begg's test (clinical effect: $z=0.86, P=0.392$; uric acid: $z=0.94$,
$P=0.346$ ) and Egger's test (clinical effect: $t=1.00, P=0.325$; uric acid: $t=0.46, P=0.650$ ) also indicated no statistically significant publication bias.

\section{Discussion}

Our current study analyzed data from 28 RCTs involving 2237 patients that aimed to assess the therapeutic effect and safety of acupuncture for gouty arthritis. Based on the findings of our study, acupuncture could further improve the clinical effective rate and decrease uric acid and VAS score when compared with western medicine. The risk of adverse events was significantly lower in acupuncture group. Regarding the decrease in CRP and ESR, the results remain debatable.

The application of different acupuncture modalities by different investigators can greatly affect curative effect of acupuncture therapy [48]. The acupuncture procedures should be performed according to syndromes differentiation based on TCM theory. In the selected studies, acupuncture intervention was administered alone [22, 31, 32, 35-37, 41, $46]$ or in combination with other therapies, which included Chinese herbal medicine [20, 21, 23, 25-27, 29, 30, 33, 44], acupoint injection [24, 47], local blocking therapy [28], bloodletting [34, 38, 42], infrared irradiation [43], moxamoxibustion [45], and western medicine [39, 40]. Regardless of the type of the additional therapy, acupuncture therapy was mainly received in experimental groups. The acupoint selection was inconsistent among included studies; nevertheless, Sp6, St36, and Ashi point were the most commonly used acupoint. In our present study, data was combined without differentiating acupoint selection and acupuncture techniques. Thus, the results indicate an overall clinical efficacy and definite conclusion could not be drawn.

Gouty arthritis is one of the most common indications for which patients seek complementary and alternative medicine treatment, even though the use of CAM was relatively low, compared with reported rates of between $28 \%$ and $90 \%$ in 


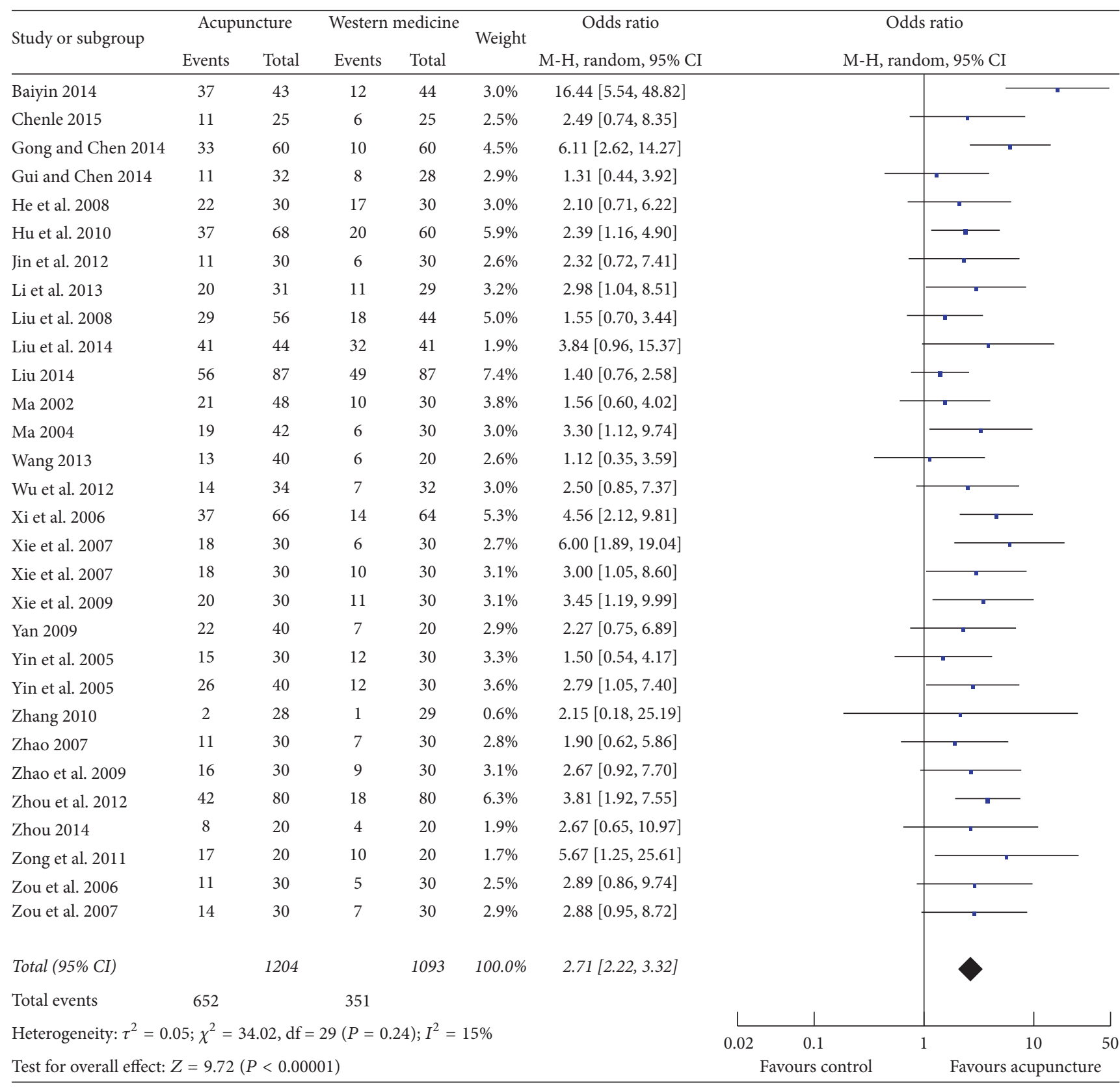

FIgURE 4: Forest plot of acupuncture therapy versus western medicine: clinical effect.

patients with rheumatoid arthritis (RA) and more than $80 \%$ in those with osteoarthritis (OA) $[49,50]$. The goal of therapy in an acute gout attack is prompt and safe termination of pain and inflammation [51]. Our results suggest that acupuncture therapy could further improve pain when compared with western medicine, but, in terms of inflammation attenuation, the results remain debatable.

When treating gouty arthritis one needs to treat acute attacks and lower excess stores of uric acid to achieve dissolution of monosodium urate crystals through a longterm reduction of serum uric acid concentrations far beyond the threshold for saturation of urate and provide prophylaxis to prevent acute flares [52]. In addition to the improvement in pain-relief and clinical effect, acupuncture therapy could further decrease uric acid, compared with conventional western pharmacological therapy. The underlying mechanism of this urate-lowering effect was still unclear, which needs to be further investigated. To analyze the possible source of heterogeneity within these studies, metaregression was performed. The type of acupuncture therapy was proved to be significantly correlated with the heterogeneity; the evidence thus suggests that the urate-lowering effects of manual 


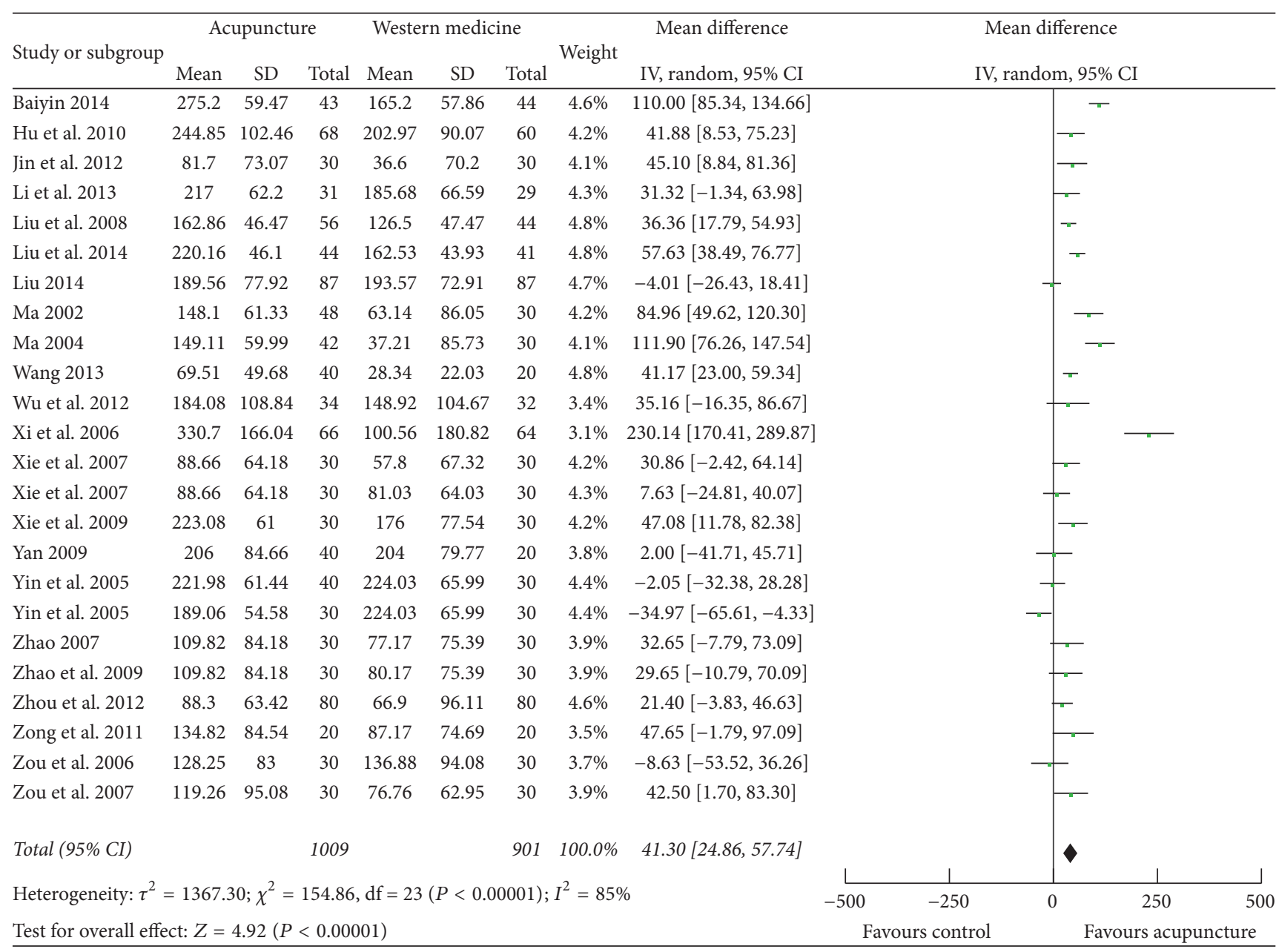

FIGURE 5: Forest plot of acupuncture therapy versus western medicine: uric acid.

acupuncture and electroacupuncture are somewhat different. No conclusions can be drawn as to which acupuncture type is superior for patients with gouty arthritis.

A previous systematic review [13] consisting of ten RCTs reported that acupuncture alone was more effective in the improvement of pain and uric acid when compared with standard western medicine. However, only ten studies were identified to be eligible; only VAS score and uric acid were analyzed as outcome. No data about the safety of acupuncture therapy was provided. In addition, two records listed in Figure 1A of the previous systematic review were miscalculated, although this would not influence the final conclusion drawn by their work [13]. Based on five required domains (study limitations, consistency, directness, precision, and publication bias), Shekelle and colleagues assessed the strength of evidence for the conclusions drawn by the aforementioned systematic review [53]. The strength of evidence for conclusions was judged to be insufficient to support or refute the effectiveness of acupuncture on symptomatic outcomes, partially due to the unreported publication bias. Our meta-analysis managed to summarize all published RCTs to compare the clinical effect and safety of acupuncture with those of western medicine. 28 RCTs were identified; clinical effect, VAS score, uric acid, ESR, CRP, and adverse events were combined; risk of bias was independently assessed by two experienced reviewers using Cochrane's tool. The results of metaregression showed a significant correlation between acupuncture type and heterogeneity in uric acid; publication bias assessment indicates no obvious publication bias. Regarding the safety of acupuncture, which is an obvious advantage of CAM therapies, the overall incidence of adverse events in acupuncture groups was significantly lower than that in western medicine group.

There are several limitations in our study. First, the methodological qualities of included studies were judged to be poor; details about allocation concealment were not described by any study, which might limit the value of conclusion about the clinical efficacy and safety of acupuncture. Second, all included studies utilized western medicine as control treatment, making the blinding of participants impossible. A sham acupuncture control is preferable, as opposed to medication or no intervention. Also, the majority of selected studies were written in Chinese, which limits the dissemination of scientific researches on acupuncture. Future 


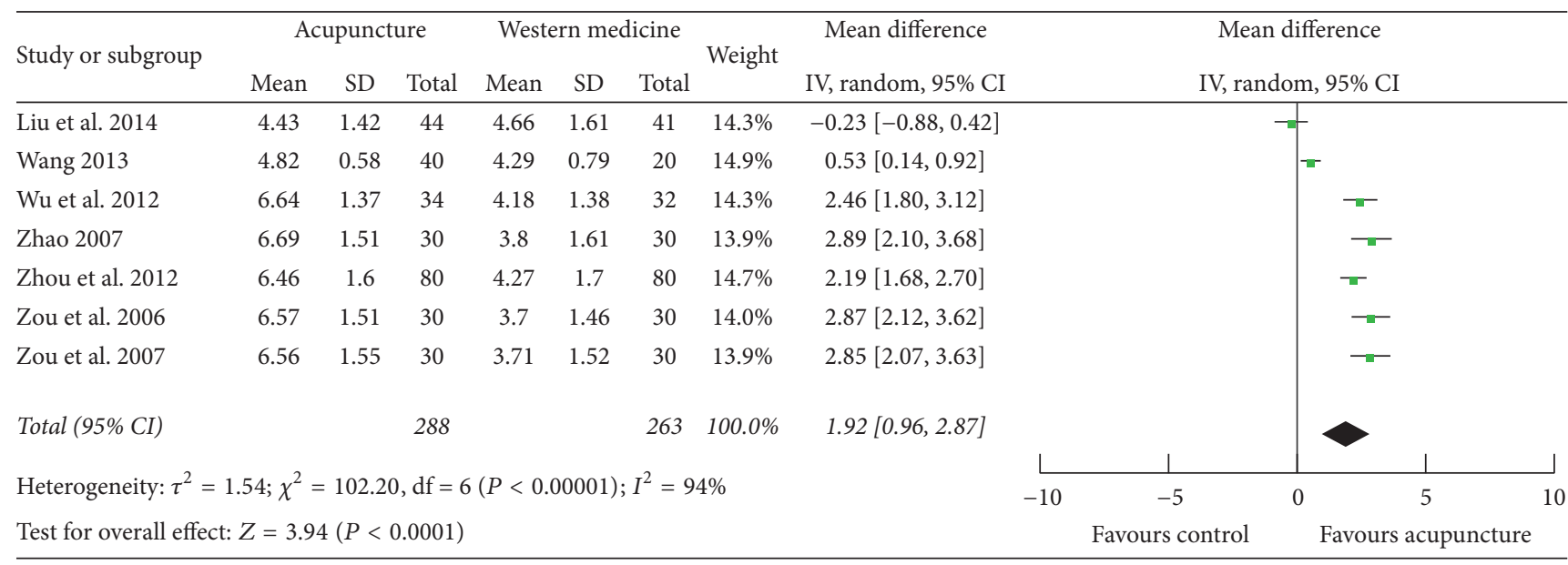

FIGURE 6: Forest plot of acupuncture therapy versus western medicine: pain intensity (VAS score).

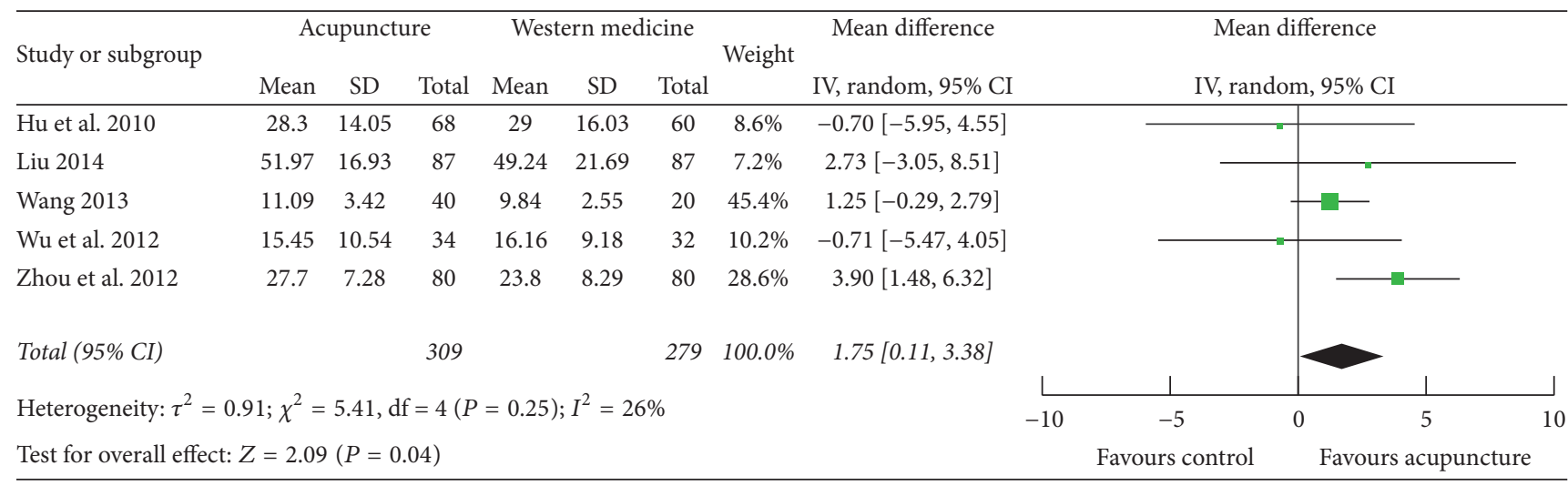

FIGURE 7: Forest plot of acupuncture therapy versus western medicine: ESR.

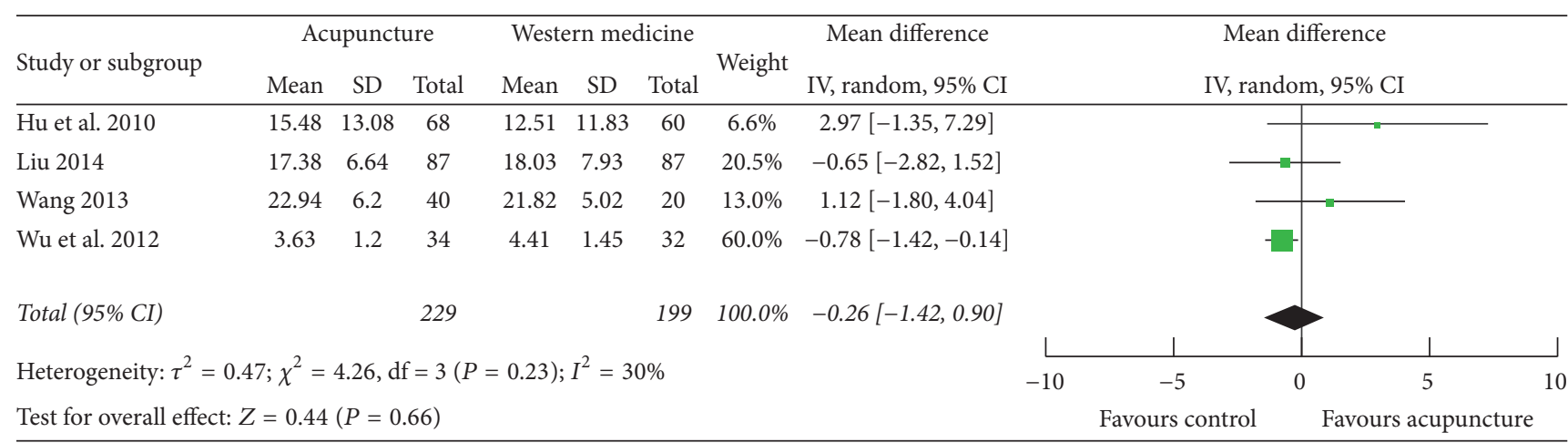

FIGURE 8: Forest plot of acupuncture therapy versus western medicine: CRP.

studies within western context are required. Third, financial considerations are important for consumers choosing CAM options as expensive treatments are generally avoided and cost is a frequent reason to stop treatment [54]. In Chinese culture, the safety and cost-effectiveness of acupuncture therapy could assure the compliance of patients. Considering participants' opinions, cost-effectiveness of acupuncture also meant its efficacy. However, only ten studies recorded adverse events associated with acupuncture and routine care; no study included cost-effectiveness assessment. Last, according to the ACR guideline, choice of pharmacologic agents should be based upon number of joints involved and pain intensity; the established pharmacologic ULT should be continued without interruption. However, some of our included studies only administered first-line therapy options (NSAIDs, corticosteroids, and colchicine); ULT therapy during acute 


\begin{tabular}{|c|c|c|c|c|c|c|c|c|c|c|}
\hline \multirow{3}{*}{$\begin{array}{l}\text { Study or subgroup } \\
\text { Gui and Chen } 2014\end{array}$} & \multicolumn{2}{|c|}{ Acupuncture } & \multicolumn{2}{|c|}{ Western medicine } & \multirow{2}{*}{ Weight } & \multirow{2}{*}{$\begin{array}{c}\text { Odds ratio } \\
\mathrm{M}-\mathrm{H} \text {, random, } 95 \% \mathrm{CI}\end{array}$} & \multirow{2}{*}{\multicolumn{4}{|c|}{$\begin{array}{c}\text { Odds ratio } \\
\text { M-H, random, 95\% CI }\end{array}$}} \\
\hline & \multirow{2}{*}{$\frac{\text { Events }}{1}$} & \multirow{2}{*}{$\begin{array}{c}\text { Total } \\
32\end{array}$} & \multirow{2}{*}{$\begin{array}{c}\text { Events } \\
15\end{array}$} & \multirow{2}{*}{$\begin{array}{c}\text { Total } \\
28\end{array}$} & & & & & & \\
\hline & & & & & $14.0 \%$ & $0.03[0.00,0.23]$ & & - & & \\
\hline He et al. 2008 & 0 & 30 & 13 & 30 & $9.4 \%$ & $0.02[0.00,0.38]$ & & & & \\
\hline Hu et al. 2010 & 0 & 68 & 4 & 60 & $9.2 \%$ & $0.09[0.00,1.74]$ & & & - & \\
\hline Jin et al. 2012 & 0 & 30 & 0 & 30 & & Not estimable & & & & \\
\hline Li et al. 2013 & 0 & 31 & 6 & 29 & $9.3 \%$ & $0.06[0.00,1.07]$ & & & & \\
\hline Liu et al. 2014 & 0 & 44 & 2 & 41 & $8.6 \%$ & $0.18[0.01,3.81]$ & & & & \\
\hline Liu 2014 & 1 & 87 & 6 & 87 & $13.9 \%$ & $0.16[0.02,1.33]$ & & & & \\
\hline Wang 2013 & 0 & 40 & 8 & 20 & $9.3 \%$ & $0.02[0.00,0.34]$ & $\leftarrow$ & & & \\
\hline Xie et al. 2009 & 0 & 30 & 14 & 30 & $9.5 \%$ & $0.02[0.00,0.33]$ & - & & & \\
\hline Yan 2009 & 4 & 40 & 2 & 20 & $16.8 \%$ & $1.00[0.17,5.98]$ & & & - & \\
\hline Total (95\% CI) & & 432 & & 375 & $100.0 \%$ & $0.08[0.03,0.23]$ & & & & \\
\hline Total events & 6 & & 70 & & & & & & & \\
\hline Heterogeneity: $\tau^{2}=$ & $87 ; x^{2}=$ & $2.20, \mathrm{~d}$ & $=8(P=0$ & 4); $I^{2}=$ & $34 \%$ & & \llcorner & $\perp$ & $\frac{1}{10}$ & $\underset{1000}{ل}$ \\
\hline Test for overall effec & $Z=4.68$ & $P<0.0$ & 001) & & & & Fav & puncture & Favours & \\
\hline
\end{tabular}

FIGURE 9: Forest plot of acupuncture therapy versus western medicine: adverse events.

TABLE 3: Metaregression of basic characteristics of trials and improvement of uric acid and VAS score.

\begin{tabular}{lccccc}
\hline Outcome & Number of RCTs & Factor tested & $t$ & $P$ & Adjusted $R^{2}$ \\
\hline \multirow{3}{*}{ Uric acid } & \multirow{2}{*}{22} & Acupuncture type & 2.21 & 0.038 & $20.20 \%$ \\
& & Combined therapy & 0.35 & 0.730 & $-6.31 \%$ \\
& & Duration of treatment & 1.58 & 0.127 & $7.67 \%$ \\
VAS score & \multirow{2}{*}{7} & Acupuncture type & -1.62 & 0.165 & $23.55 \%$ \\
& & Combined therapy & 1.32 & 0.243 & $12.20 \%$ \\
& & Duration of treatment & -1.27 & 0.259 & $10.11 \%$ \\
\hline
\end{tabular}

Note. RCT: randomized controlled trial; VAS: visual analogue scale.

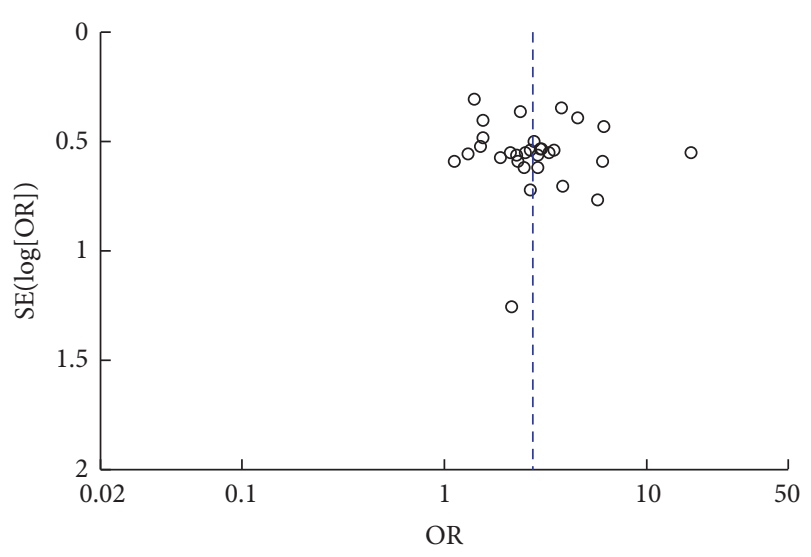

FIGURE 10: Funnel plot of acupuncture therapy versus western medicine: clinical effect.

attack was stopped; some studies only used ULT, without using first-line pharmacologic options. This would lead to an exaggeration of conclusions; thus, the effect of intervention should be interpreted cautiously.

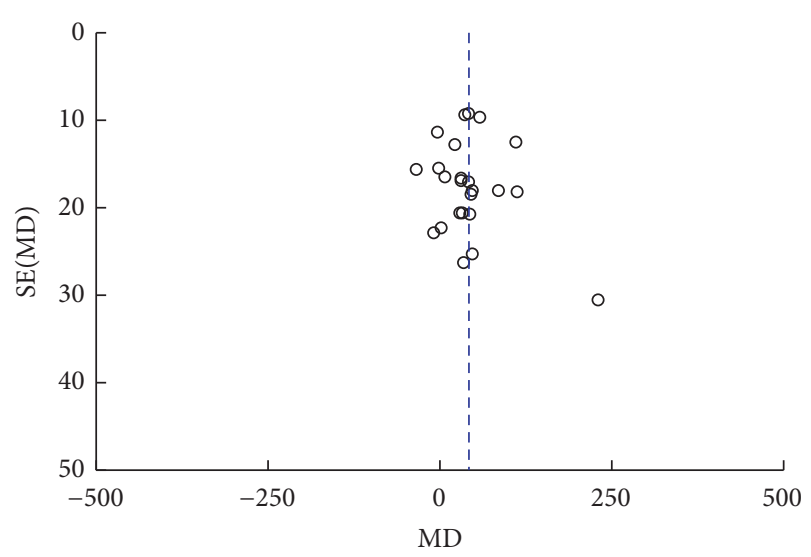

FIGURE 11: Funnel plot of acupuncture therapy versus western medicine: uric acid.

In summary, the findings of our current study suggest that acupuncture is better in improving clinical effective rate, decreasing uric acid, and VAS score compared with western medicine. The overall incidence of adverse events in 
acupuncture group was lower than that in western medicine group. Due to the methodological deficiency of included trials, acupuncture therapy could not be guaranteed as standard CAM treatment. Additional RCTs with rigorous design and larger sample size are encouraged.

\section{Competing Interests}

The authors have declared no conflict of interests.

\section{References}

[1] P. Richette and T. Bardin, "Gout," The Lancet, vol. 375, no. 9711, pp. 318-328, 2010.

[2] R. L. Wortmann, "Gout and hyperuricemia," Current Opinion in Rheumatology, vol. 14, no. 3, pp. 281-286, 2002.

[3] P. Ghosh, M. Cho, G. Rawat, P. A. Simkin, and G. C. Gardner, "Treatment of acute gouty arthritis in complex hospitalized patients with anakinra," Arthritis Care and Research, vol. 65, no. 8, pp. 1381-1384, 2013.

[4] E. Arromdee, C. J. Michet, C. S. Crowson, W. M. O'Fallon, and S. E. Gabriel, "Epidemiology of gout: is the incidence rising?" Journal of Rheumatology, vol. 29, no. 11, pp. 2403-2406, 2002.

[5] P. P. Khanna, G. Nuki, T. Bardin et al., "Tophi and frequent gout flares are associated with impairments to quality of life, productivity, and increased healthcare resource use: results from a cross-sectional survey," Health and Quality of Life Outcomes, vol. 10, article 117, 2012.

[6] E. Pascual, "Persistence of monosodium urate crystals and low-grade inflammation in the synovial fluid of patients with untreated gout," Arthritis \& Rheumatism, vol. 34, no. 2, pp. 141$145,1991$.

[7] J. A. Singh and V. Strand, "Gout is associated with more comorbidities, poorer health-related quality of life and higher healthcare utilisation in US veterans," Annals of the Rheumatic Diseases, vol. 67, no. 9, pp. 1310-1316, 2008.

[8] B. F. Mandell, "Clinical manifestations of hyperuricemia and gout," Cleveland Clinic Journal of Medicine, vol. 75, supplement 5, pp. S5-S8, 2008.

[9] N. Dalbeth, B. Clark, K. Gregory et al., "Mechanisms of bone erosion in gout: a quantitative analysis using plain radiography and computed tomography," Annals of the Rheumatic Diseases, vol. 68 , no. 8, pp. 1290-1295, 2009.

[10] M. Hamburger, H. S. B. Baraf, T. C. Adamson III et al., "2011 Recommendations for the diagnosis and management of gout and hyperuricemia," Postgraduate medicine, vol. 123, no. 6, pp. 3-36, 2011.

[11] D. Khanna, P. P. Khanna, J. D. Fitzgerald et al., "2012 American College of Rheumatology guidelines for management of gout. Part 2: therapy and antiinflammatory prophylaxis of acute gouty arthritis," Arthritis Care \& Research, vol. 64, no. 10, pp. 14471461, 2012.

[12] S. L. Kolasinski, "Food, drink, and herbs: alternative therapies and gout," Current Rheumatology Reports, vol. 16, no. 4, article 409, 2014.

[13] W. B. Lee, S. H. Woo, B.-I. Min, and S.-H. Cho, "Acupuncture for gouty arthritis: a concise report of a systematic and metaanalysis approach," Rheumatology, vol. 52, no. 7, pp. 1225-1232, 2013.
[14] D. Moher, A. Liberati, J. Tetzlaff, D. G. Altman, and P. Group, "Preferred reporting items for systematic reviews and metaanalyses: the PRISMA statement," PLoS Medicine, vol. 6, no. 7, Article ID e1000097, 2009.

[15] S. L. Wallace, H. Robinson, A. T. Masi, J. L. Decker, D. J. McCarty, and T. F. Yü, "Preliminary criteria for the classification of the acute arthritis of primary gout," Arthritis \& Rheumatism, vol. 20, no. 3, pp. 895-900, 1977.

[16] I. Peláez-Ballestas, C. H. Cuevas, R. Burgos-Vargas et al., "Diagnosis of chronic gout: evaluating the american college of rheumatology proposal, European league against rheumatism recommendations, and clinical judgment," Journal of Rheumatology, vol. 37, no. 8, pp. 1743-1748, 2010.

[17] H. J. E. M. Janssens, J. Fransen, E. H. van de Lisdonk, P. L. C. M. van Riel, C. van Weel, and M. Janssen, "A diagnostic rule for acute gouty arthritis in primary care without joint fluid analysis," Archives of Internal Medicine, vol. 170, no. 13, pp. 11201126, 2010.

[18] S. A. O. T. C. Medicine, Standards for Diagnosis of Syndromes or Diseases of TCM and Evaluation of the Therapeutic Effect, Nanjing University Press, 1994.

[19] J. P. T. Higgins and S. Green, Cochrane Handbook for Systematic Reviews of Interventions, Version 5.1.0, The Cochrane Collaboration, 2011.

[20] M. Baiyin, "Clinical observation on Mongolian medicine in the treatment of gout," Journal of North Pharmacy, vol. 11, no. 9, pp. 36-37, 2014.

[21] M. Chenle, "Clinical observation on mongolian medicine combined with silver needle in the treatment of 25 cases of gout," Journal of Medicine and Pharmacy of Chinese Minorities, no. 5, pp. 7-8, 2015.

[22] Y. Gong and M. Chen, "Clinical study of fire needling plus abdominal acupuncture in the treatment of acute gouty arthritis," Hubei Journal of Traditional Chinese Medicine, vol. 36, no. 12, p. 62, 2014.

[23] M. Gui and M. Chen, "Acupuncture combined with Chinese herbal medicine for 32 cases of acute gouty arthritis," Hunan Journal of Traditional Chinese Medicine, vol. 30, no. 12, pp. 7475, 2014.

[24] Y. He, L. Gu, X. Li, and X. Yang, "Electroacupuncture plus acupoint injection for the treatment of acute gouty arthritis," Modern Journal of Integrated Traditional Chinese and Western Medicine, vol. 17, no. 3, pp. 383-384, 2008.

[25] Y. Hu, J. Li, K. Cao et al., "Clinical observation on acupuncture combined with traditional Chinese medicine for treating acute gouty arthritis of 68 cases," Journal of Sichuan of Traditional Chinese Medicine, vol. 28, no. 5, pp. 72-74, 2010.

[26] Z. Jin, S. Jiang, Y. Wang et al., "Therapeutic observation on combined use of acupuncture and medication for acute gouty arthritis," Shanghai Journal of Acupuncture and Moxibustion, vol. 31, no. 9, pp. 670-672, 2012.

[27] J. Li, T. He, X. Yan, and X. Du, "Combined use of acupuncture and medication for damp-heat with blood stasis acute gouty arthritis," Asia-Pacific Traditional Medicine, vol. 9, no. 2, pp. 8788, 2013.

[28] B. Liu, H.-M. Wang, and F.-Y. Wang, "Observation on therapeutic effect of electroacupuncture combined with local blocking therapy on acute gouty arthritis," Chinese Acupuncture and Moxibustion, vol. 28, no. 9, pp. 659-661, 2008.

[29] J. Liu, W. Xu, and X. Zhu, "Clinical observation on fire needling plus external application of Chinese herbal medicine 
for the treatment of acute gouty arthritis," Shanghai Journal of Acupuncture and Moxibustion, vol. 33, no. 4, pp. 343-345, 2014.

[30] Z. Liu, "Combined use of acupuncture and medication for acute gouty arthritis with retention of damp-heat," Shanxi Journal of Traditional Chinese Medicine, vol. 30, no. 4, pp. 27-28, 2014.

[31] X. Ma, "Acupuncture treatment of gouty arthritis and effect on serum uric acid," Chinese Acupuncture and Moxibustion, vol. 22, no. 3, pp. 151-152, 2002.

[32] X. Ma, "Clinical analysis for the acupuncture treatment in cases of gouty renal damage," Journal of Traditional Chinese Medicine, vol. 24, no. 3, pp. 185-187, 2004.

[33] H. Wang, "Clinical observation on acupuncture combined with four wonderful decoction for acute gouty arthritis," Chinese Journal of Basic Medicine in Traditional Chinese Medicine, vol. 39, no. 6, pp. 688-689, 2013.

[34] Y. Wu, M. Xu, S. Zheng, J. Liao, C. Hu, and W. Zheng, "Clinical research on electroacupuncture combined with blood-letting therapy by fire needling for treating acute gouty arthritis," Liaoning Journal of Traditional Chinese Medicine, vol. 39, no. 7, pp. 1400-1402, 2012.

[35] Y. Xi, T. Zou, Z. Ai, Y. Liu, and Q. Zhang, "Clinical observation on acupuncture treatment of acute gouty arthritis in terms of clearing heat eliminating dampness and stasis," Acupuncture Research, vol. 31, no. 6, pp. 362-364, 2006.

[36] J.-Y.Xie, L. Wang, Q.-X. Li, and X.-M. Li, "Study on mechanisms of electroacupuncture treatment of acute gouty arthritis," Chinese Acupuncture and Moxibustion, vol. 27, no. 12, pp. 898-900, 2007.

[37] X.-Q. Xie, Y.-X. Cao, F. Li, Y. Meng, and L. Deng, “Observation on therapeutic effect of surrounded needling therapy on acute gouty arthritis," Chinese Acupuncture and Moxibustion, vol. 29, no. 5, pp. 375-377, 2009.

[38] B. Yan, "Observation on therapeutic effect of acupuncture combined with blood-letting therapy on gout arthritis," Journal of Kunming Medical University, vol. 30, no. 12, pp. 101-104, 2009.

[39] Y. Yin, H. Zhang, and T. Zhang, "Clinical observation on electroacupuncture combined with medicine for treatment of acute gouty arthritis," Chinese Acupuncture and Moxibustion, vol. 25, no. 10, pp. 683-685, 2005.

[40] J. Zhang, "Observation on therapeutic effects of eyeacupuncture therapy for acute gouty arthritis," Liaoning Journal of Traditional Chinese Medicine, vol. 37, no. 2, pp. 334-335, 2010.

[41] H. Zhao, "Combined use of acupuncture and medication for the treatment of 60 cases of acute gouty arthritis," Journal of Clinical Acupuncture and Moxibustion, vol. 23, no. 9, pp. 26-27, 2007.

[42] Q.-W. Zhao, J. Liu, X.-D. Qu et al., "Observation on therapeutic effect of electroacupuncture plus blood-letting puncture and cupping combined with diet intervention for treatment of acute gouty arthritis," Chinese Acupuncture and Moxibustion, vol. 29, no. 9, pp. 711-713, 2009.

[43] L. Zhou, Q. Xu, and W. Zhang, "Comparative observation of therapeutic effects of acupuncture combined with infrared irradiation and western medicine on acute gouty arthritis," World Journal of Acupuncture-Moxibustion, vol. 22, no. 1, pp. 30-34, 2012.

[44] M. Zhou, "Combined use acupuncture and medication for acute gouty arthritis of 20 cases," Nei Mongol Journal of Traditional Chinese Medicine, no. 14, p. 44, 2014.

[45] J. Zong, Y. Gao, S. Wang, H. He, and J. Zheng, "40 cases of acute gouty arthritis treated by warming needle moxibustion," Journal of Sichuan of Traditional Chinese Medicine, vol. 29, no. 3, pp. 115$117,2011$.

[46] R. Zou, H.-X. Zhang, T.-F. Zhang, and Y. Xu, "Treatment to acute gouty arthritis with electro-acupuncture at different frequencies versus medication," Chinese Journal of Clinical Rehabilitation, vol. 10, no. 43, pp. 188-189, 2006.

[47] R. Zou, H.-X. Zhang, T.-F. Zhang, and Y. Xu, "Observation on therapeutic effect of electroacupuncture combined with acupoint-injection on acute gouty arthritis," Chinese Acupuncture and Moxibustion, vol. 27, no. 1, pp. 15-17, 2007.

[48] Z. T. Lv, W. Song, J. Wu et al., "Efficacy of acupuncture in children with nocturnal enuresis: a systematic review and meta-analysis of randomized controlled trials," Evidence-Based Complementary and Alternative Medicine, vol. 2015, Article ID 320701, 12 pages, 2015.

[49] E. Ernst, "Complementary and alternative medicine in rheumatology," Best Practice and Research: Clinical Rheumatology, vol. 14, no. 4, pp. 731-749, 2000.

[50] P. Katz and F. Lee, "Racial/ethnic differences in the use of complementary and alternative medicine in patients with arthritis," Journal of Clinical Rheumatology, vol. 13, no. 1, pp. 3-11, 2007.

[51] N. Schlesinger, "Management of acute and chronic gouty arthritis: present state-of-the-art," Drugs, vol. 64, no. 21, pp. 2399-2416, 2004.

[52] N. Schlesinger, N. Dalbeth, and F. Perez-Ruiz, "Gout-what are the treatment options?" Expert Opinion on Pharmacotherapy, vol. 10, no. 8, pp. 1319-1328, 2009.

[53] P. G. Shekelle, J. FitzGerald, S. J. Newberry et al., Management of Gout, Agency for Healthcare Research and Quality, Rockville, Md, USA, 2016.

[54] J. K. Rao, K. Kroenke, K. A. Mihaliak, S. C. Grambow, and M. Weinberger, "Rheumatology patients' use of complementary therapies: results from a one-year longitudinal study," Arthritis Care \& Research, vol. 49, no. 5, pp. 619-625, 2003. 


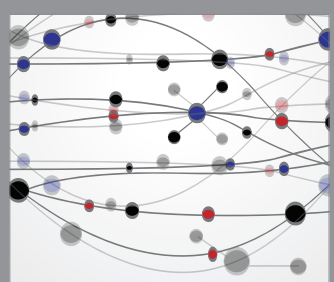

The Scientific World Journal
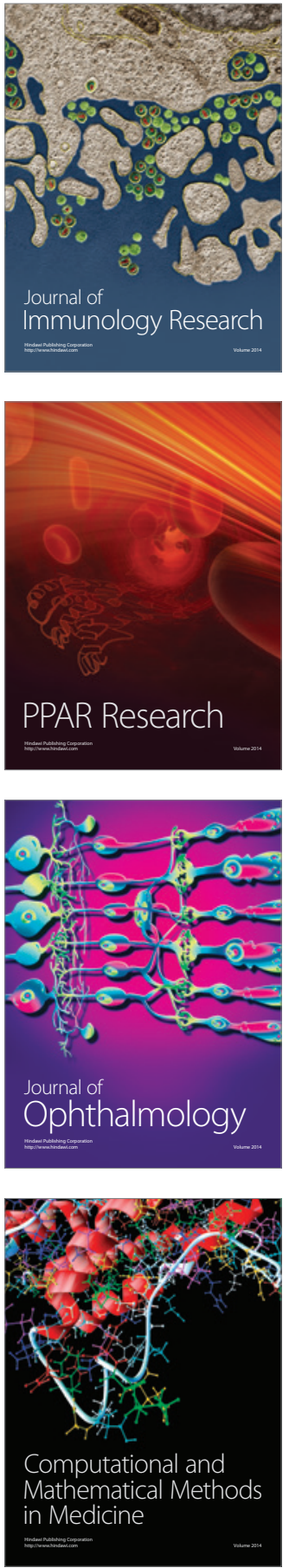

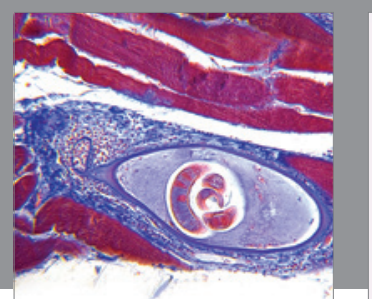

Gastroenterology Research and Practice

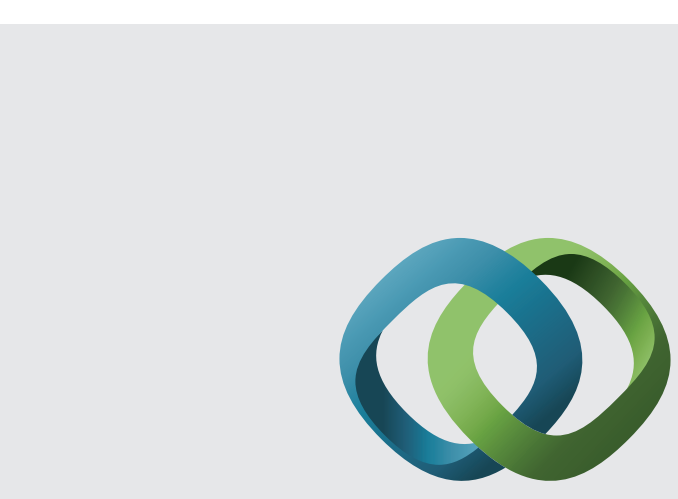

\section{Hindawi}

Submit your manuscripts at

http://www.hindawi.com
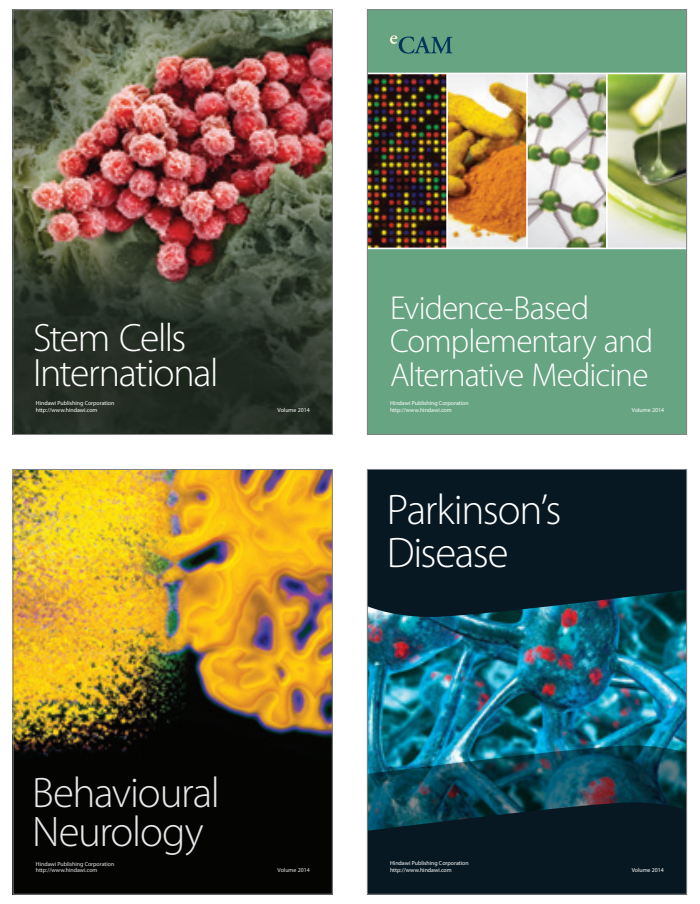
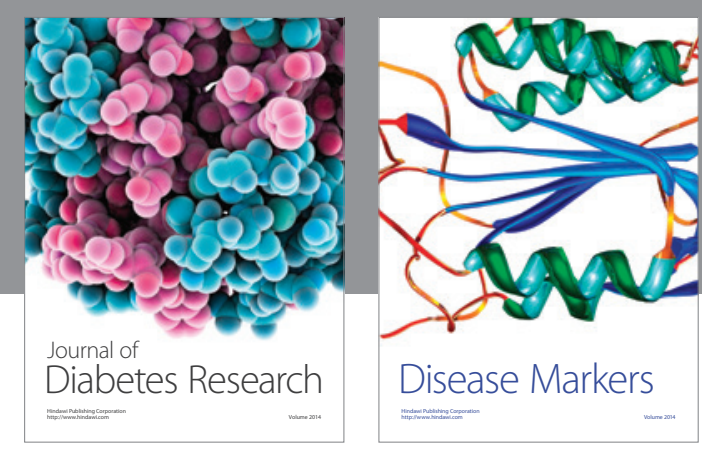

Disease Markers
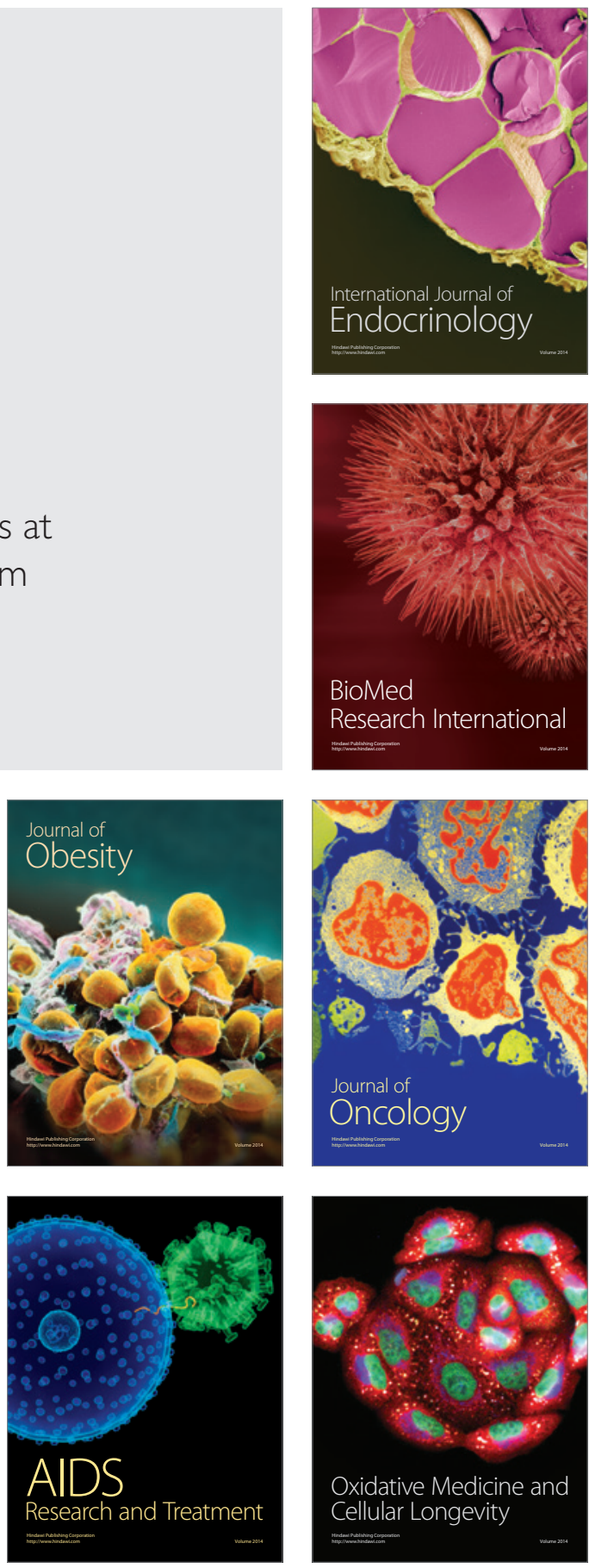